\title{
PUBLICATIONS
}

\section{FUNGAL TECHNOLOGY}

The Filamentous Fungi, Volume 4. Fungal Technology. Edited by J.E. Smith, D. R. Berry, and B. Kristiansen. Pp. 400. ISBN 0-7131-2857-7 (Edward Arnold Publishers Ltd.: Baltimore, MD: 1983) $\$ 74.50$.

a recent years, taxomomists have seen fit to elevate fungi into a separate kingdom, on equal footing with green plants and multicellular animals. This new status reflects the unique characteristics the group has always possessed: chitinous, threadlike cell walls, assimilative growth, unusual biosynthetic pathways, and the ability to degrade certain recalcitrant substrates and to thrive at lower moisture levels than most microorganisms.

Filamentous fungi grow by penetrating their substrates with a mass of branching filaments, the mycelium. Degradative enzymes are secreted across the hyphal wall into the substrate; nutrients broken down by the extracellular enzymes are absorbed and assimilated back into the mycelium. As long as the food supply is plentiful the assimilative phase continues and the fungus grows vegetatively. When food becomes limiting, two events may occur: the fungus may sporulate, and ofien, simultaneously, may initiate a characteristic secondary metabolism. During secondary metabolism, the fungus produces species-specific natural products with diverse chemical structures. The best known fungal sccondary metabolites are pharmacologically active and include penicillin, cephalosporin, lysergic acids, and aflatoxin. Sometimes, when growth is inhibited in the presence of an abundant carbon source, fungal metabolism becomes "deranged" and large quantities of normal intermediates may accumulate. The citric acid fermentation of Aspergillus niger is the prototype of an efficient industrial process based on this principle.

I'he series on The Filamentous Fungi is a semi-encyclopedic review of the remarkable attributes of molds as tools for basic research and for industry. 'The fourth volume, Fungal Technology, serves as a companion to the first, Industrial Mycology. Because the theme is technology, the volume emphasizes the engineering problems of relating molds to machines. Other aspects of fungal biotechnology such as lignin and cellulose degradation, fungal control of insects, and mycotoxins are also covered.

Fungal properties have been harnessed for human benefit since antiquity. The domestic arts of leavening, brewing, wine making, cheese ripening, and other such fermentations are well known in the West. Dr. Steinkraus (Dept. Food Science and Technology, N.Y. Agricultural Experiment Station, Geneva, NY) discusses the mold fermentations of the Orient. Soy sauce and sake (rice wine) are only two of a large number of Eastern foods that require filamentous fungi. The author describes both household and pilot plant versions of meat analogues from Rhizopus-fermented soybeans (tempe kedele) and a versatile condiment from Aspergillus-fermented soybean-grain mixtures (miso). Tempe burgers are now available in California, and miso is gaining popularity in the "health food" culture.

Industrial mycology and its associated biotechnology are analogous to modern agriculture, in which a monoculture of fungi is grown for human benefit. Instead of soil there are substrates, instead of fertilizer there are "appropriate" nutrients, and instead of fields there are fermentors. The harvest consists of enzymes, organic acids, antibiotics, and many specialized pharmaceuticals.

I he filamentous growth form and the preference for non-aqueous environments impose special problems for large-scale, liquid, industrial fermentation. Oxygen concentration must be kept high, and valves must be specially designed to prevent blockage by mycelium. The book reviews process control, principles of mass and energy transfer, industrial scaleup, and instrumentation from an engineering standpoint. 'The chapter on instrumentation (D. S. Flyrin, H. R. M. Research Institute, High Wycombe, Berkshire, U.K.) is a welcome essay that points out that microelectronic: is not the universal solution to all instrumentation and control problems: it is easier to measure temperature than glucose concentration, and the complex nature of fermentation processes demands complex assays and implementation.
Two topics, sometimes ignored in engineering-oriented works, are particularly well covered: culture preservation and mutagenesis. Strain degeneration remains a problematic and mysterious phenomenon in industrial mycology. A. H. S. Onions (Commonwealth Mycological Institute, Kew, Surrey, U.K.) gives an excellent discussion of methods for preservation of fungal cultures. The chapter "Industrial Fungal Genetics and Strain Selection" by R. I. Rowlands (Beecham Pharmaceuticals, Worthing, West Sussex, U.K.) also deserves special commendation. Industrial strain improvement has proceeded largely through a combination of "brute force" mutagenesis and selection. Rowlands has succeeded in combining theories about mulagenesis, mutagen specificity, and mutator genes with the pragmatics of application in "the real world"-no small feat.

It has become commonplace in reviews of this sort to speak of the problems associated with multi-authored books. Perhaps we forget the advantages. In a well-edited, well-integrated work such as this, a group of experts joins to give up-to-date, comprchensive coverage of each topic. 'The series on The Filamentous Fungi belongs in every research library, as well as on the shelves of individual scientists who do applied work with molds and who are affluent enough to afford the book. Volume 4 is a welcome addition that matches the standards of the preceding volumes.

Joan Bennett, Ph.D., is Professor of Biology, Tulane University, New Orleans, LA.

\section{BIOTECHNOLOGY PATENT REVIIW}

Biotechnology Patents (1981-1982). Microbiological Syntheses. Recent Advances. Biotechnology Review No. 1 . Edited by S. Torrey. Pp.338. ISBN 0. 8155-0937-5 (Noyes Data Corp.: Park Ridge, NJ:1983) \$48.00.

The subject matter of this volume 1 is drawn from over 300 patents granted in the United States between 